\title{
Pion and Kaon Structure Functions at an Electron-Ion Collider
}

\author{
Tanja Horn ${ }^{* \dagger}$ \\ Catholic University of America and Jefferson Lab \\ E-mail: horntecua.edu
}

Pions and kaons are, along with protons and neutrons, the main building blocks of nuclear matter. They are connected to the Goldstone modes of dynamical chiral symmetry breaking, the mechanism thought to generate all hadron mass in the visible universe. The distribution of the fundamental constituents, the quarks and gluons, is expected to be different in pions, kaons, and nucleons. However, experimental data are sparse. As a result, there has been persistent doubt about the behaviour of the pion's valence quark structure function at large Bjorken- $x$ and virtually nothing is known about the contribution of gluons. The Electron-Ion Collider with an acceptance optimized for forward physics could provide access to structure functions over a larger kinematic region. This would allow for measurements testing if the origin of mass is encoded in the differences of gluons in pions, kaons, and nucleons, and measurements that could serve as a test of assumptions used in the extraction of structure functions and the pion and kaon form factors. Measurements at an EIC would also allow to explore the effect of gluons at high $x$.

XXV International Workshop on Deep-Inelastic Scattering and Related Subjects

3-7 April 2017

University of Birmingham, $U K$

\footnotetext{
* Speaker.

${ }^{\dagger}$ Supported in part by NSF grants PHY1306227 and PHY1306418
} 


\section{Introduction}

Pions and kaons occupy a special role in nature [1] and thus have a central role in our current description of nucleon and nuclear structure. The pion is the lightest quark system, with a single valence quark and a single valence antiquark. It is also the particle responsible for the long range character of the strong interaction that binds the atomic nucleus together. A general belief is that the rules governing the strong interaction are left-right, i.e. chirally, symmetric. If this were true, the pion would have no mass. The chiral symmetry of massless Quantum Chromodynamics (QCD) is broken dynamically by quark-gluon interactions and explicitly by inclusion of light quark masses, giving the pion and kaon mass. The pion and kaon are thus seen as the key to confirm the mechanism that dynamically generates nearly all of the mass of hadrons and central to the effort to understand hadron structure.

Experimental knowledge of the partonic structure of the pion is very limited due to the lack of a stable pion target. Our current knowledge of the pion structure function in the valence region is obtained primarily from pionic Drell-Yan scattering [2, 3, 4], and in the pion sea region at low Bjorken- $x$, from hard diffractive processes measured on $e-p$ collisions at HERA [5]. These data seem to indicate that the pion sea has approximately one-third of the magnitude of the proton sea, while from the parton model one expects the pion sea to be two-thirds of the proton sea.

The distribution of the fundamental constituents, the quarks and gluons, is expected to be different in pions, kaons, and nucleons. However, as discussed above experimental data on the pion structure function are sparse and there are no data on the kaon structure function to date. As a consequence of the lack of pion data there has been persistent doubt about the behaviour of the pion's valence quark structure function at large Bjorken- $x$ and virtually nothing is known about the contribution of sea quarks and gluons [6]. The Electron-Ion Collider (EIC) with an acceptance optimized for forward physics has the potential for accessing pion and kaon structure functions over a large kinematic region through the Sullivan process [7]. This would allow for measurements testing if the origin of mass is encoded in the differences of gluons in pions, kaons, and nucleons [8], and measurements that could serve as a test of assumptions used in the extraction of structure functions and the pion and kaon form factors.

\section{Experimental Considerations}

In the Sullivan process, one measures the contribution to the electron Deep Inelastic Scattering (DIS) of the meson cloud of a proton target. An immediate consequence is that the nucleon parton distributions contain a component which can be attributed to the meson cloud. Note that in the Sullivan process, the mesons in the nucleon cloud are virtual (off-shell) particles. For the process to provide reliable access to a meson target, the pole associated with that meson must be the dominant process. Recent calculations [9] estimate the impact of off-shellness in the Bethe-Salpeter Equation/Dyson-Schwinger Equation (BSE/DSE) framework. It was found that the meson can be considered on-shell for pions up to $t<0.6 \mathrm{GeV}^{2}$ and for kaons up to $t<0.9 \mathrm{GeV}^{2}$. This suggests that pion and kaon structure functions can be accessed through the Sullivan process.

To experimentally determine the off-shellness correction, one can use a procedure similar to that used for corrections in studies of the neutron in deuterium. There, interactions in the medium 

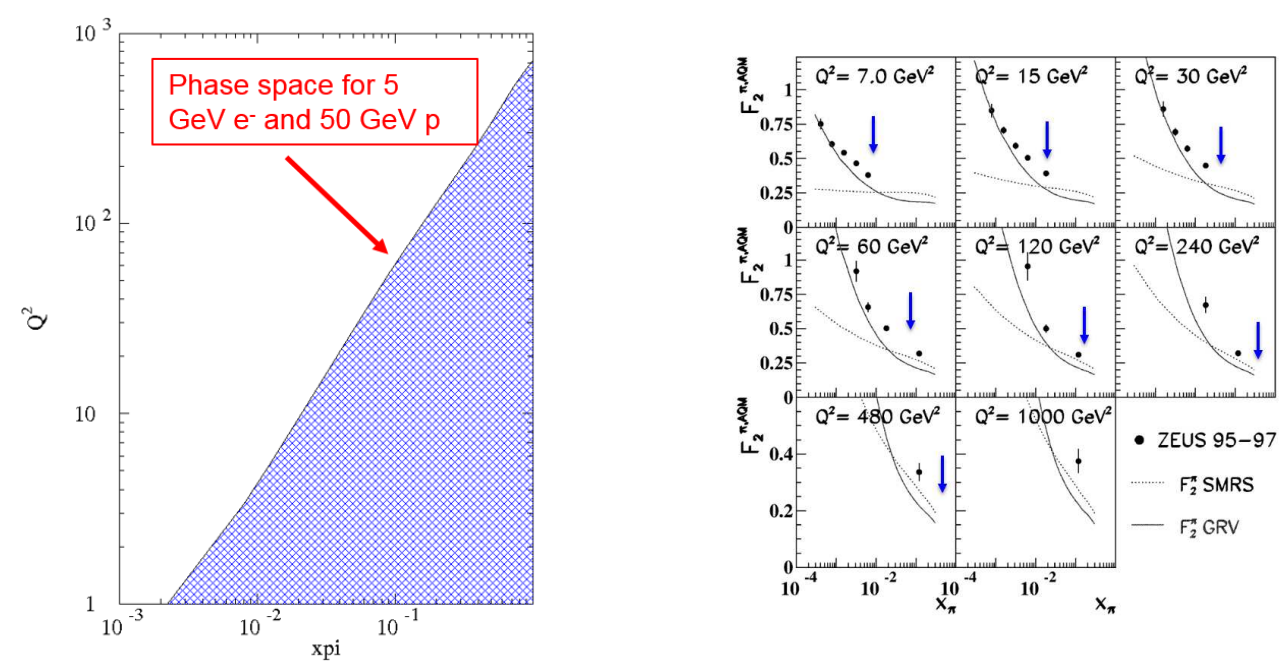

Figure 1: EIC kinematic reach.

have to be taken into account, which is done through nuclear binding corrections. By binning in $t$ up to the projected on-shell/off-shell limit, an off-shellness correction could be determined in a similar fashion.

\section{Pion and Kaon Structure Functions at an EIC}

The Electron-Ion Collider will provide a large $\left(x, Q^{2}\right)$ landscape for both pion and kaon structure measurements. The acceptance at the EIC is ideal for structure function measurements, essentially covering the full forward region and also covering much higher $x$ than previous measurements. The projections in the present report assume roughly a year of running, which corresponds to 26 weeks at $50 \%$ efficency and a geometric detection efficiency for forward going particles at small $t$ of 20\%. The EIC kinematic reach in $x$ is shown in Fig. 1 for electron and proton beam energies of $5 \mathrm{GeV}$ and $50 \mathrm{GeV}$, at a luminosity of $10^{34} \mathrm{~s}^{-1} \mathrm{~cm}^{-2}$. The kinematic coverage reaches down to $x_{\pi, K}=0.01$ or even smaller values for both pion and kaon. The small $x_{\pi, K}$ region will be constrained by structure functions obtained at HERA. The blue arrows in Fig. 1 indicate the $x_{\text {min }}$ values for future EIC data at the same $Q^{2}$ values as the HERA data.

The projections of kaon structure functions were obtained from those of the pion case by scaling with the coupling constants of Ref. Kroll11 and the geometric detection efficiencies. The resulting kaon structure function projections are of roughly similar quality as the pion structure functio projections for small $t$, forward particle detection acceptances of JLEIC. Detector similations have shown that the decay particles from $\Lambda$ decay can be distinguished from the beam particles near the 7 th or 8 th downstream dipole. Fig. 2 shows the kaon structure function projections for beam energies of $5 \mathrm{GeV}$ electrons and 100 (left) $\mathrm{GeV}$ and 50 (right) $\mathrm{GeV}$ protons as a function of $Q^{2}$ and for different values of $x$, at a luminosity of $100 \mathrm{fb}^{-1}$. To constrain gluons the $Q^{2}$ evolution is important. As one can see, for small ranges in $x$ the $Q^{2}$ evolution may not be sufficient, however, and other techniques may need to be explored to supplement. 

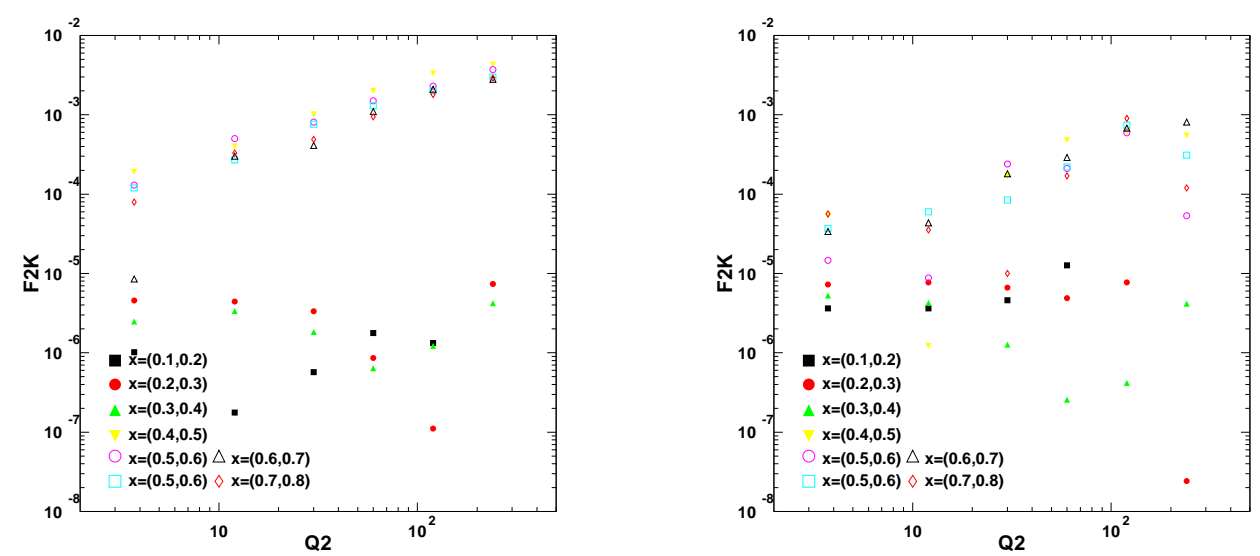

Figure 2: Kaon structure function projections for beam energies of $5 \mathrm{GeV}$ electrons and 100 (left) $\mathrm{GeV}$ and 50 (right) $\mathrm{GeV}$ protons as a function of $Q^{2}$ and for different values of $x$. A luminosity of $100 \mathrm{fb}^{-1}$ is assumed.
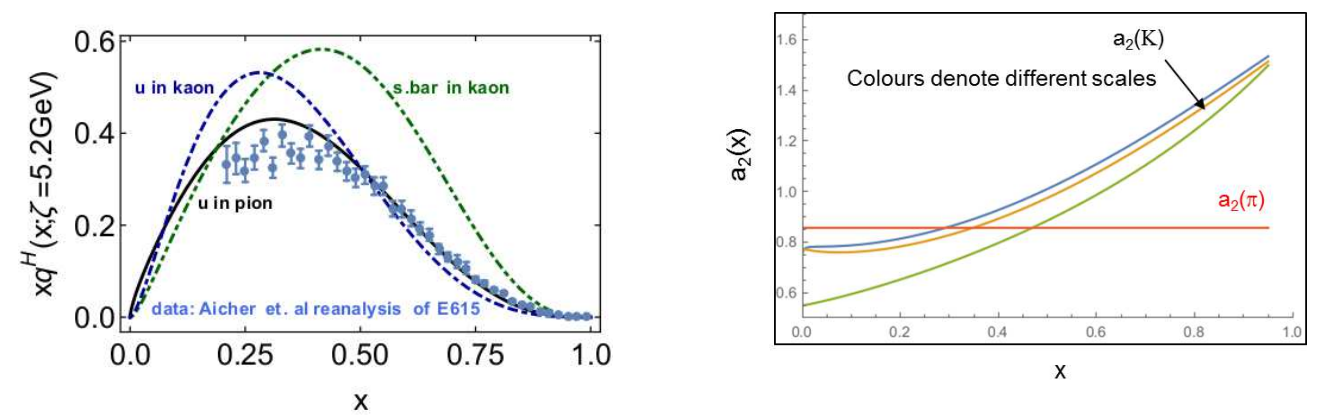

Figure 3: (a) DSE Parton Distribution Functions from Ref. [11]; (b) $a_{2 \pi}(x)$ and $a_{2 K}(x)$ at different scales.

\section{Towards Flavour Decomposition}

Parity-violating probes may provide a way to make progress towards a full flavour decomposition. These include neutral- and charged-current probes. Observables from charged-current processes can be obtained through a comparison of electron versus positron interactions. Neutralcurrent processes include measurements of the $x F_{3}$ nucleon structure functions ( $\gamma Z$ interference contribution) and the neutral-current conserving parity-violating asymmetry, which can be expressed through the coefficients $a_{2 \pi}(x)$ and $a_{2 K}(x)$. Fig. 3(a) shows the DSE parton distributions from Ref. [11]. One can see that the valence quarks in the kaon carry much more of the kaon's momentum than is stored in the pion's valence-quarks. Fig. 3(b) shows the resulting coefficients $a_{2 \pi}$ and $a_{2 K}$ as a function of $x$. The different colours denote different scales, e.g., $a_{2 K}$ at the hadronic scale $\zeta H=0.51$ (green curve), $a_{2 K}$ at scale $\zeta=2 \mathrm{GeV}$ (green curve), and $a_{2 K}$ at scale $\zeta=5.2 \mathrm{GeV}$ (blue curve). At $x=1, a_{2 K}$ is independent of the scale, while at $x=0 a_{2 K}$ approaches $9 / 5-\sin ^{2} \theta_{W}$ because all valence-quark PDFs become identical at $x=0$ under evolution. Simulation studies to project the feasibility of such measurements at EIC are currently ongoing. 


\section{Summary}

In summary, nucleons and the lightest mesons, pons and kaons, are the basic building blocks of nuclear matter. We should know their structure functions. The distribution of quarks and gluons in pions, kaons, and nucleons is expected to be different. Measurements of the structure functions at low to moderate $x$ could shed light on the question if the origin of mass is encoded in differences of gluons in pions, kaons and nucleons (at non-asymptotic values of $Q^{2}$ ). Using electroweak processes, e.g., through parity-violating probes or neutral vs. charged-current interactions, disentangling flavour dependence seems possible.

\section{References}

[1] T. Horn and C.D. Roberts, J. Phys. 43 (2016) no. 7, 073001.

[2] J.S. Conway et al., Phys. Rev. D 39 (1989) 39.

[3] J. Badier et al., Z. Phys. C18 (1983) 281.

[4] B. Betev et al., Z. Phys. C28 (1985) 9.

[5] H1 Collaboration, C. Adloff et al., Eur. Phys. J. C6 (1999) 587; V. Andreev et al., arxiv:1312.4821.

[6] R.J. Holt, C.D. Roberts, Rev. Mod. Phys. 82, 2991 (2010).

[7] J.D. Sullivan, Phys. Rev. D5 (1972) 1732.

[8] C.D. Roberts, Few Body Syst. 58 (2017) no.1, 5.

[9] S.-X. Qin, C. Chen, C. Mezrag, C.D. Roberts, arXiv:1702:06100 (2017).

[10] S. V. Goloskokov and P. Kroll, Eur. Phys. J. A 47, 112 (2011) [arXiv:1106.4897 [hep-ph]].

[11] C. Chen, L. Chang, C.D. Roberts, S. Wang, H.-S. Zong, Phys. Rev. D 93 (2016) no.7, 074021. 